\title{
Concurrent Management of Bilateral Ureteropelvic Junction Obstruction in Children Using Robotic-Assisted Laparoscopic Surgery
}

\author{
Drew A. Freilich, Hiep T. Nguyen, Joseph Borer, Caleb Nelson, Carlo C. Passerotti \\ Department of Urology, Children's Hospital Boston, Harvard Medical School, Boston, \\ Massachusetts, USA
}

\begin{abstract}
Introduction: Bilateral ureteropelvic junction (UPJ) obstruction occurs infrequently. When surgical management is deemed necessary, staged pyeloplasties traditionally have been recommended to minimize the morbidity associated with performing procedures concurrently. With the advent of robotic-assistance, concurrent surgical management can more readily be performed laparoscopically. In this report, we evaluated the safety and outcome of managing patients with bilateral UPJ obstruction with concurrent robotic-assisted laparoscopic pyeloplasty.

Materials and Methods: We performed a retrospective review of five patients with bilateral ureteropelvic junction obstruction who underwent concurrent bilateral robotic-assisted pyeloplasties at our institution between October 2003 and April 2007. Technical consideration for patient positioning, robotic set-up, port placement, and the use of a hitch stitches was assessed. The operative time, complications, analgesic needs, length of hospitalization, and overall success of the procedure were evaluated.

Results: Operative time ranged from 235 to 541 minutes (mean = 384). Estimated blood loss was 5-100 $\mathrm{cc}(\mathrm{mean}=48.0)$. Length of hospitalization ranged from 1.3 to 3.6 days (mean $=2.4)$. Ureteral stents were removed 3-8 weeks postoperatively. There were no complications. All kidneys demonstrated decreased hydronephrosis on postoperative ultrasound or improved drainage parameters on diuretic renography or IVP.

Conclusions: Simultaneous bilateral robotic-assisted laparoscopic pyeloplasties utilizing 4-port access is feasible and safe. It provides an effective method of managing patients with bilateral UPJ obstruction, avoiding the burden and morbidity of performing staged surgeries.
\end{abstract}

Key words: pediatrics; hydronephrosis; laparoscopy; robotics; pyeloplasty

Int Braz J Urol. 2008; 34: 198-205

\section{INTRODUCTION}

In children, bilateral ureteropelvic junction obstruction is present in approximately $10-40 \%$ of UPJ obstructions $(1,2)$. Most bilateral cases are asymmetrical, with one side being more severely affected than the other. When surgical intervention is deemed necessary, staged pyeloplasties traditionally have been recommended. While the success of performing concurrent bilateral open pyeloplasties has been reported (3), many surgeons remain hesitant to perform this procedure because of the morbidity associated with operating on both kidneys concurrently and the potential for acute bilateral renal obstruction. 
As a result, staged pyeloplasty is often considered safer. However, it requires the need for the patient to undergo two separate operations, which are separated by a potentially prolonged recovery period.

Laparoscopic surgery has achieved increasing popularity in the management of ureteropelvic junction (UPJ) obstruction in children. Its less invasive nature provides for more rapid recovery and improved cosmesis. Successful concurrent bilateral pyeloplasties utilizing free-hand laparoscopy have been reported (4). However, it remains technically challenging. Robotic-assisted surgery has significantly decreased surgeon learning curves as compared to free-hand laparoscopy, while at the same time achieving postoperative outcomes comparable to open pyeloplasty (5). Thus, it may be of benefit in the concurrent surgical management of bilateral UPJ obstruction. In this study, we evaluated the safety and outcome of performing concurrent robotic-assisted laparoscopic pyeloplasties.

\section{MATERIALS AND METHODS}

After Institutional Review Board approval was obtained a retrospective review was initiated. Between October 2003 and April 2007, five patients underwent concurrent robotic-assisted bilateral pyeloplasties for the management of bilateral ureteropelvic junction obstruction at our institution. All patients had preoperative radiologic imaging including ultrasonography, diuretic renography, or intravenous pyelogram indicative of the diagnosis of bilateral ureteropelvic junction obstruction. The indications for surgery included increasing degree of hydronephrosis, pain, urinary tract infection, and parental preference.

\section{Surgical Technique}

Preoperatively, patients received a clear liquid diet for 24 hours and a rectal suppository the night before the procedure. After the induction of anesthesia, the patient was placed in supine position and a 30-degree wedge was placed under the patient elevating the more affected side. The patient was then carefully secured to the operating table, prepped and draped.
To insert the trocars, the table was rotated to place the patient in a flat supine position in order for the ports to be placed safely into the peritoneum. A 12 $\mathrm{mm}$ camera port was inserted in the umbilicus. Three 5 or $8 \mathrm{~mm}$ working ports were then inserted; the first in the midline $10 \mathrm{~cm}$ above the umbilicus and the other two in the mid-clavicular line in the right and left lower quadrant (Figure-1). After placing the ports, the patient was maximally rotated to the contralateral side, which helped to shift the bowel away from the renal fossa. The robotic system was then engaged.

The procedure was performed as previously described by Lee et al. (5). In brief, the peritoneum was incised along the avascular white line of Toldt. After reflection of the colon and incising through anterior lamina of Gerota's fascia, blunt dissection was performed to expose the renal pelvis, UPJ and proximal ureter. A hitch stitch was utilized to elevate the renal pelvis for easier dissection and suturing (Figure-2). An incision through the renal pelvis was then made above the UPJ. After excising the UPJ segment, the ureter was transposed over any existing crossing vessel and anastomosed back to the pelvis after it was spatulated. A running suture of 5-0 vicryl or monocryl was used in all cases. A kidney internal splint/stent (KISS) or a double-J stent was placed, depending on the surgeon's preference.

After completion of the anastomosis, surgery was performed on the contralateral kidney. All the ports were wrapped in sterile towels to maintain sterility during the changeover. Either of two methods was used to set up the robotic system for the contralateral pyeloplasty. The first involved moving the robot to the opposite side, which necessitated powering down the robot and re-arranging the room set up. The second involved rotating the patient 180 -degrees, with careful monitoring of the endotracheal tube and the patient during the change of position. Over time, we preferred the later method, which was more practical. Careful discussion and planning with the anesthesiologists (such as placement of extension tubing and longer monitoring cables) prior to start of the procedure allowed the patients to be moved safely and efficiently during the changeover. The exact same set-up and surgical procedure was performed as previously described. At the end of the procedure, all ports sites were closed. 


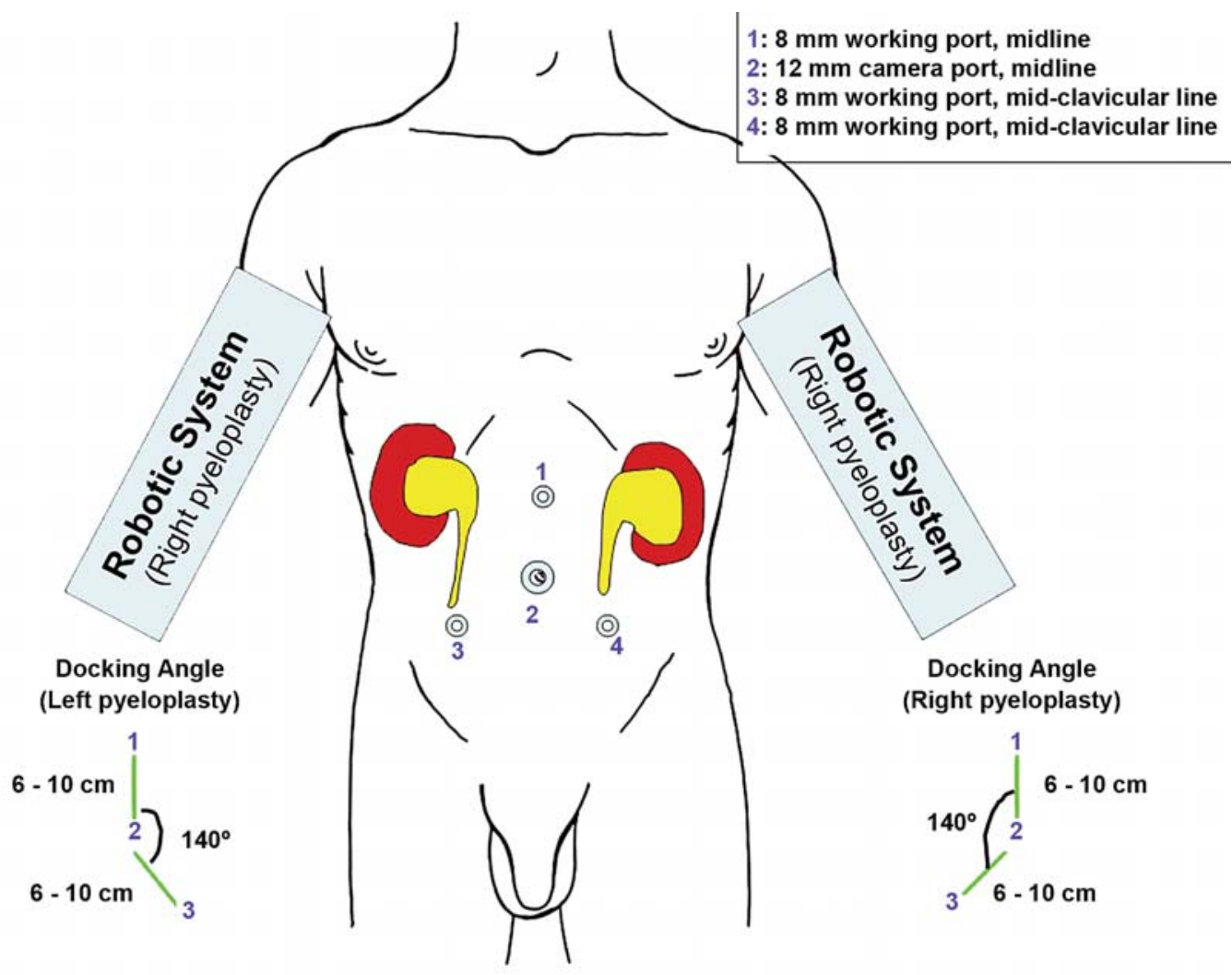

Figure 1 - Surgical cart and port placement.

The stents were removed per the surgeon's preference. Follow-up imaging with renal ultrasonography were performed on all patients. If improvement in the degree of hydronephrosis was observed postoperatively and the patients were asymptomatic, only an ultrasonography was performed during subsequent follow-up visits. If there were any concerns regarding the degree of hydronephrosis observed postoperatively, a diuretic renography (MAG-3) or intravenous pyelogram was carried out to assess drainage.

\section{RESULTS}

The age of the patients was 3.4-14.0 years (mean - 9.5 years). All patients had a voiding cystouretrogram (VCUG) preoperatively to rule out concomitant reflux. No patient had preoperative drainage (i.e. nephrostomy). The operative time ranged from
235 to 541 minutes $($ mean $=384)$. Estimated blood loss ranged from 5 to $100 \mathrm{cc}(0.2-2 \mathrm{cc} / \mathrm{kg})$ with a mean of $48.0 \mathrm{cc}(1.3 \mathrm{cc} / \mathrm{kg})$. Total perioperative morphine

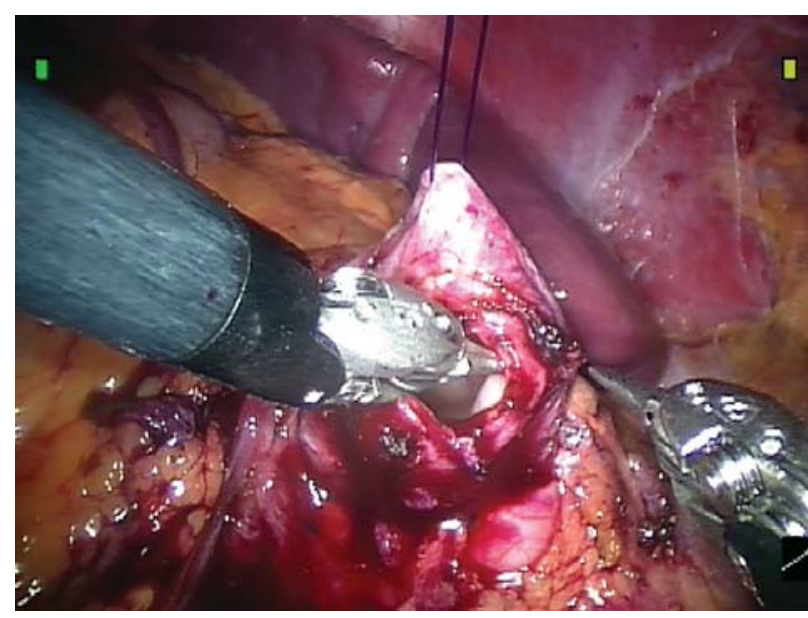

Figure 2 - Renal pelvis hitch stitch to facilitate exposure and anastomosis with the ureter. 
equivalent requirement (i.e. codeine and fentanyl) ranged from 0.77 to $3.71 \mathrm{mg} / \mathrm{kg}($ mean $=1.7)$. There were no intraoperative or postoperative complications. The length of hospitalization ranged from 1.3 to 3.6 days (mean $=2.4)$. All patients had ureteral stents placed bilaterally ( 3 with double-J stents, 2 with KISS). In 4 patients, the stents were removed sequentially 3 to 6 weeks postoperatively, with a 1-2 weeks interval in between each stent removal. In one patient, both stents were removed simultaneously at 8 weeks. All patients received prophylactic antibiotics until both stents were removed. Postoperative follow-up ranged from 2 to 43.7 months (mean =11.4). Table- 1 details the patients' intraoperative findings, conditions and radiologic evaluation at the time of last follow-up. Figure-3 illustrates typical pre- and postoperative radiologic findings in our patients with bilateral UPJ obstruction managed with concurrent laparoscopic pyeloplasties.

Table 1 - Patient description.

\begin{tabular}{|c|c|c|c|}
\hline Patient & Preoperative Findings & Etiology & Follow-up Radiology \\
\hline 1 & $\begin{array}{c}\text { Presentation: Flank pain and nausea. } \\
\text { Renogram: Left T } 1 / 212 \text { min with } 40 \% \\
\text { residual. Right t } 1 / 278 \text { min with } 76 \% \\
\text { residual }\end{array}$ & $\begin{array}{l}\text { Right crossing vessel. } \\
\text { Left intrinsic stenosis }\end{array}$ & $\begin{array}{l}41 \text { months: US - Bilateral mild } \\
\text { pelvicaliectasis. } \\
\text { Patient asymptomatic }\end{array}$ \\
\hline 2 & $\begin{array}{l}\text { Presentation: Flank pain and nausea. } \\
\text { US: Bilateral moderate to severe hydro- } \\
\text { nephrosis }\end{array}$ & Bilateral crossing vessel & $\begin{array}{l}9 \text { months: US - Right side with } \\
\text { mild pelvicaliectasis. Left side } \\
\text { with moderate pelvicaliectasis. } \\
\text { Patient asymptomatic }\end{array}$ \\
\hline 3 & $\begin{array}{l}\text { Presentation: Flank pain and hematuria. } \\
\text { US: Left moderate hydronephrosis. Right } \\
\text { moderate to severe hydronephrosis }\end{array}$ & Bilateral intrinsic stenosis & $\begin{array}{l}6 \text { months: Renogram - Renal } \\
\text { function: Left } 51 \% \text {, Right } 48 \% \text {. } \\
\text { Bilateral delay in spontaneous } \\
\text { collecting drainage. Complete } \\
\text { drainage after furosemide without } \\
\text { obstruction bilaterally. Patient } \\
\text { asymptomatic. }\end{array}$ \\
\hline 4 & $\begin{array}{l}\text { Presentation: Hydronephrosis after bilat- } \\
\text { eral megaureter repair. } \\
\text { US: Severe bilateral hydronephrosis }\end{array}$ & Bilateral crossing vessel & $\begin{array}{l}6 \text { months: IVP - Right side with } \\
\text { considerable calyceal dilation. } \\
\text { Left side with residual pelvicaliec- } \\
\text { tasis. Prompt drainage bilaterally. } \\
\text { Patient asymptomatic }\end{array}$ \\
\hline 5 & $\begin{array}{l}\text { Presentation: Pyelonephritis. } \\
\text { US: Left moderate hydronephrosis. } \\
\text { Severe right hydronephrosis. Renogram: } \\
\text { Left T } 1 / 2>100 \text { min with } 87 \% \text { residual. } \\
\text { Right T } 1 / 255 \text { min with } 70 \% \text { residual }\end{array}$ & $\begin{array}{l}\text { Bilateral crossing vessels. } \\
\text { Right secondary intrinsic } \\
\text { obstruction }\end{array}$ & $\begin{array}{l}2 \text { months: Left minimal pelvicali- } \\
\text { ectasis. Right no hydronephrosis. } \\
\text { Patient asymptomatic }\end{array}$ \\
\hline
\end{tabular}

$I V P=$ intravenous pyelography; $U S=$ ultrasonography. 
A
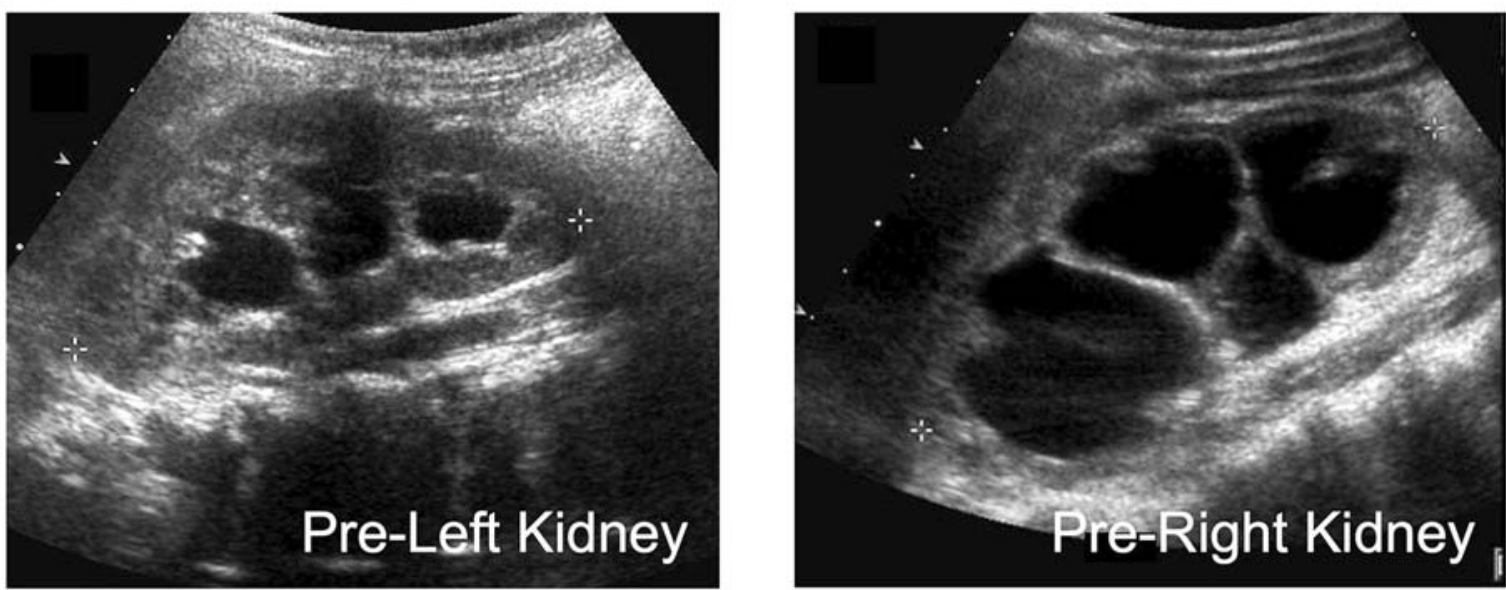

B

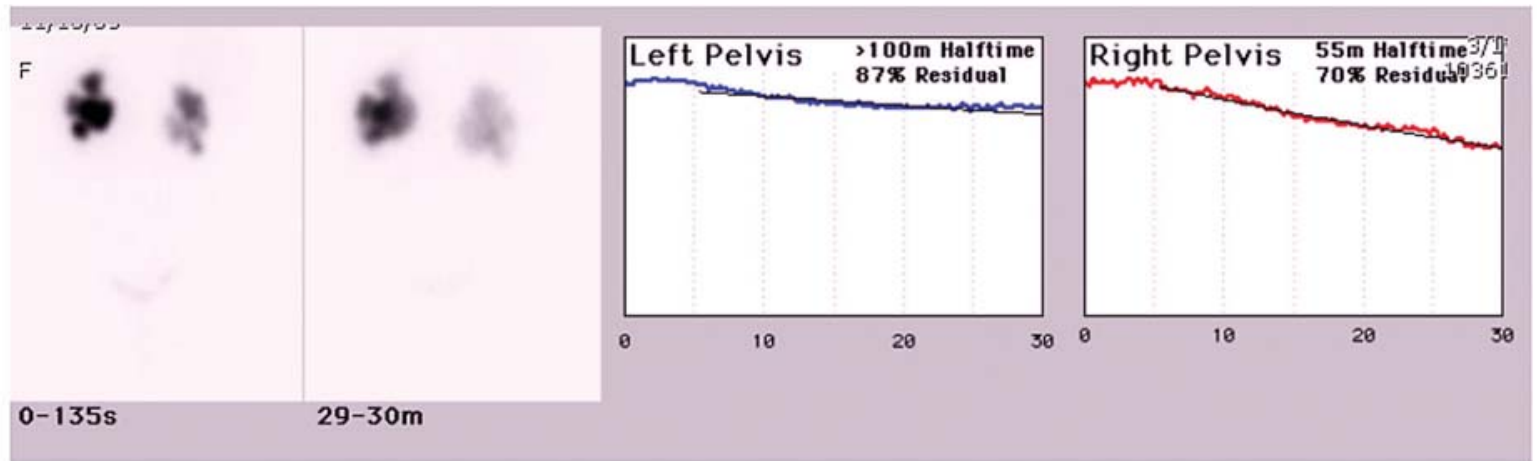

C
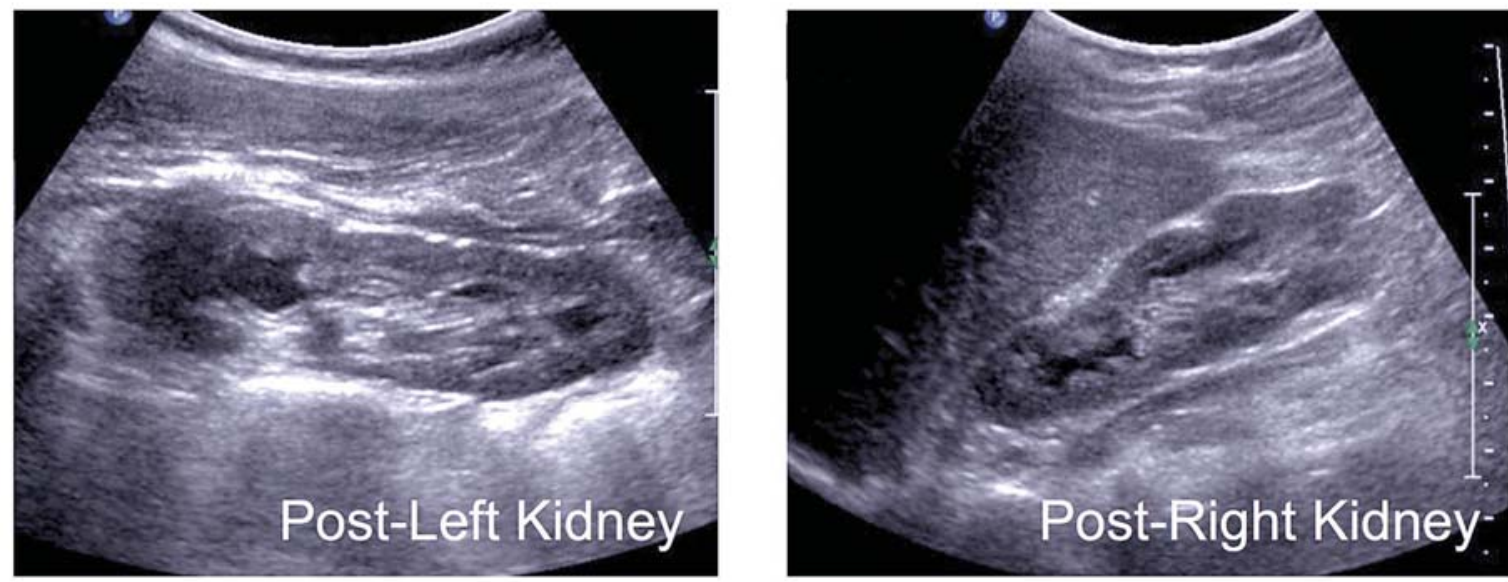

Figure 3 - Ultrasound findings. A) Preoperative ultrasound reveals moderate hydronephrosis of the left and severe hydronephrosis of the right kidney. B) Preoperative diuretic MAG-3 renogram demonstrating significant residual of the radionuclide in both kidneys after furosemide administration. C) Postoperative ultrasound demonstrates no hydronephrosis in the right kidney and minimal pelvicaliectasis in the left kidney 2 months after surgery. 


\section{COMMENTS}

The efficacy and safety of robotic-assisted unilateral pyeloplasty is well documented. To our knowledge this is the first reported series regarding concurrent robotic-assisted laparoscopic bilateral pyeloplasties. We found that performing these procedures concurrently can be time saving. The operative time was reasonable, considering our mean time for performing a unilateral robotic laparoscopic pyeloplasty was 219 minutes (5). The most experienced robotic surgeon at our institution performed his first bilateral robotic-assisted case in 235 minutes, compared to 268 minutes in a published series utilizing free-hand laparoscopy. With increased experience we believe the time savings of simultaneous bilateral robotic-assisted pyeloplasty versus free-hand laparoscopy will continue to increase (5). We observed that by re-positioning the patient rather than the robotic system we could decrease the changeover time to 10-20 minutes. In addition, we decreased the operative times by inserting all 4 trocars at the beginning of the surgery, avoiding the need to place new ports when operating on the contralateral kidney. This also helped to decrease the operative times by avoiding the need to change around the robotic instruments to pass and cut sutures, provide suction/ irrigation, and remove materials.

We also found that this procedure may have significant benefits compared to staged open procedure. The use of perioperative morphine equivalent in this case series (mean of $1.7 \mathrm{mg} / \mathrm{kg}$ ) is only marginally higher than a recently published cohort from our institution who underwent unilateral open pyeloplasty (mean $1.5 \mathrm{mg} / \mathrm{kg}$ ). Since the patients in this series only underwent one procedure rather two, this suggests that concurrent bilateral pyeloplasties subjected patients to less pain than staged unilateral surgical interventions. The mean length of hospitalization (2.4 days) for patients undergoing bilateral laparoscopic pyeloplasties is identical to those undergoing unilateral laparoscopic pyeloplasty and is less than that for open surgery (3.5 days) (5). Of note, the length of hospitalization consistently decreased over time, presumably secondary to increased surgeon experience with robotic-assisted surgery in unilateral pyeloplasty procedures and the postoperative monitoring of such patients. Additionally, the patient benefits from improved cosmesis associated with minimally invasive surgery. Most importantly, the entire procedure with all the necessary re-positioning maneuvers was done without any intraoperative complications. However, future larger scale studies with long term patient follow up will be required to completely evaluate the safety of concurrent robotic-assisted laparoscopic bilateral pyeloplasty as compared to a staged approach.

Moreover, our short-term follow up data suggests that concurrent robotic-assisted bilateral laparoscopic pyeloplasties may have postoperative outcomes comparable to that of unilateral open and conventional laparoscopic procedures. In follow-up, all patients in this series were asymptomatic. Their postoperative radiological evaluation demonstrated significant improvement in the degree of hydronephrosis on US or in the drainage parameters seen on diuretic renography or IVP. The level of imaging improvement in conjunction with clinical improvement was used to determine success. Stenting patterns were similar to the unilateral laparoscopic pyeloplasty cases. Placement of the stents and their staged removal avoided the potential complication of simultaneous bilateral renal obstruction. It could be argued that unlike the staged open procedure, where stenting is not routinely performed, concurrent surgery requires two additional procedures for the removal of the double-J stents. We feel however, that the additional procedures (stent removal) while requiring anesthesia when performed on children, have minimal risks and morbidity. Alternatively, KISS stents that can be removed in the clinic without anesthesia can be utilized, as was the preference of one of the surgeons in this study. At our institution, an ultrasound is performed 1-3 months after stent removal to asses the level of hydronephrosis. If significant improvement is observed, a follow-up ultrasound is performed at 612 months postoperatively. If the initial postoperative ultrasound does not show improvement, a follow up ultrasound is performed three months after the initial postoperative ultrasound. If this repeat ultrasound does not demonstrate significant improvement, a furosimide-MAG3 renogram is performed. Prospective studies regarding the impact on the quality of life of this type of surgical management will be required to more definitely assess the utility of simultaneous bilateral pyeloplasties. 


\section{CONCLUSION}

Robotic-assisted surgery offers an advantage in the management of bilateral renal pathologies such as bilateral UPJ obstruction. The results of this small cohort in conjunction with our institution's increasing experience with robotic-assisted surgery demonstrates that robotic-assisted simultaneous bilateral pyeloplasties are feasible, and may have postoperative outcomes comparable to unilateral open and conventional laparoscopic pyeloplasty. The use of the four ports, patient positioning and the hitch stitch will help to make the procedure effective and allow it to be performed in a time saving manner.

\section{ACKNOWLEDGEMENTS}

Dr. Carlo C. Passerotti received grants from CAPES and FAPESP, Brazilian Funding Agencies.

\section{CONFLICT OF INTEREST}

None declared.

\section{REFERENCES}

1. Nixon HH: Hydronephrosis in children; a clinical study of seventy-eight cases with special reference to the role of aberrant renal vessels and the results of conservative operations. Br J Surg. 1953; 40: 601-9.

2. Lebowitz RL, Griscom NT: Neonatal hydronephrosis: 146 cases. Radiol Clin North Am. 1977; 15: 49-59.

3. Eckstein HB, Drake DP: Simultaneous bilateral pyeloplasties. Proc R Soc Med. 1976; 69: 665.

4. Schwab CW 2nd, Casale P: Bilateral dismembered laparoscopic pediatric pyeloplasty via a transperitoneal 4-port approach. J Urol. 2005; 174: 1091-3.

5. Lee RS, Retik AB, Borer JG, Peters CA: Pediatric robot assisted laparoscopic dismembered pyeloplasty: comparison with a cohort of open surgery. J Urol. 2006; 175: 683-7; discussion 687.

Accepted after revision: January 18, 2008

Correspondence address:

Dr. Hiep T. Nguyen

Department of Urology

Children's Hospital Boston

300 Longwood Avenue, Hunnewell-353

Boston, MA 02115, USA

Fax: + 1617 730-0474

\section{EDITORIAL COMMENT}

In the last decade, interest has been evolving in the search for implementation of minimally invasive surgical techniques in pediatric population harboring urological pathologies. As a result of this process, the adoption, evolution and diversification of the laparoscopic approach in children has been inevitable, contributing to better esthetic results, increased magnification and improved intraoperative visualization, reduced postoperative pain, and shorter hospital stays.

Initially used as a diagnostic modality in the treatment of cryptorchidism, pediatric laparoscopic 
surgery is currently performed for complex ablative (e.g. nephrectomy, adrenalectomy, etc.) as well as reconstructive procedures such as ureteropelvic junction obstruction (UPJO). Laparoscopic pyeloplasty can be done either trans or retroperitonealy. Advocates of the retroperitoneal approach suggested an easier dissection, but there is less working space in the retroperitoneum, often making the procedure difficult in smaller children and infants, and there is some question as to whether crossing vessels are more easily missed. In addition, a bilateral retroperitoneal approach implies intraoperative repositioning of the patient and lack of possible use of common ports for both sides.

The introduction of robotic surgery could offer real advantages including a greater ability for intracorporeal suturing, enhanced stereoscopic visualization with true depth-of-field vision, and shortening of the learning curve for laparoscopy.

The study presented in this issue deals with the feasibility and safety of performing bilateral robotic-assisted laparoscopic repair of UPJO. The authors should be applauded for their contribution in popularization and diversification of robotic-assisted surgery in pediatric urology. The description of the technical approach is clear and detailed, and the use of common ports for both sides, introduced at the beginning of the procedure, is reasonable and appears to contribute to better esthetic results and decreased repositioning time. There is no doubt that feasibility and safety has been proven, however, the small number of patients and the short term follow-up do not allow concluding the late outcome of the procedure. Lessons learned from adult series have suggested that although failures become evident within the first 12 months, they can occur as late as 3 years after intervention (1). As such, pediatric patients should be followed up at least that long to ensure a lasting result. With laparoscopic pyeloplasty reported success rate of more than $90 \%$, comparable with the results of the gold standard open pyeloplasty, it is not surprising that endopyelotomy lost the game and is in course of being abandoned as a first line treatment, unless performed in very selective situations. Failures of laparoscopic pyeloplasty may sometimes occur, however infrequently and the experience gained with adults has revealed that recurrent UPJO can be endoscopically resolved, with a high success rate. The minimal invasiveness of the endoscopic approach in such cases also appears to be appealing in the pediatric population, obviating the need for another open or laparoscopic repair. It was performed successfully at any age and it should be kept in mind as a possible alternative in children (2).

Overall, the early results with robotically assisted laparoscopic pyeloplasty are encouraging and warrant further evaluation in pediatric urological surgery. It appears that the robot is most helpful to those early in their training, and its major value will be in increasing access to minimally invasive procedures in centers lacking experience in complex laparoscopic techniques. However, its cost effectiveness remained to be determined in relation to the type of procedure and the individual institutional surgical volume.

\section{REFERENCES}

1. Dimarco DS, Gettman MT, McGee SM, Chow GK, Leroy AJ, Slezak J, et al.: Long-term success of antegrade endopyelotomy compared with pyeloplasty at a single institution. J Endourol. 2006; 20: 707-12.

2. Sofer M, Binyamini J, Ekstein PM, Bar-Yosef Y, Chen J, Matzkin H, et al.: Holmium laser ureteroscopic treatment of various pathologic features in pediatrics. Urology. 2007; 69: 566-9.

Dr. Mario Sofer Director of Endourological Service Tel-Aviv Sourasky Medical Center, Tel-Aviv University Tel-Aviv, Israel E-mail:mariosofer@hotmail.com 